\title{
Protocol Based Real-Time Continuous Electroencephalography for Detecting Vasospasm in Subarachnoid Hemorrhage
}

\author{
Jeong-Ho Hong, M.D., Jae Seung Bang, M.D., ${ }^{2}$ Jin-Heon Chung, M.D., ${ }^{3}$ Moon-Ku Han, M.D., Ph.D. ${ }^{3}$ \\ Department of Neurology, ${ }^{1}$ Keimyung University Dongsan Medical Center, Daegu, Korea \\ Departments of Neurosurgery, ${ }^{2}$ Neurology, ${ }^{3}$ Seoul National University Bundang Hospital, Seongnam, Korea
}

A continuous electroencephalography (CEEG) can be helpful in detecting vasospasm and delayed cerebral ischemia in aneurysmal subarachnoid hemorrhage (SAH). We describe a patient with an aneurysmal SAH whose symptomatic vasospasm was detected promptly by using a real-time cEEG. Patient was immediately treated by intraarterial vasodilator therapy. A 50-year-old woman without any significant medical history presented with a severe bifrontal headache due to acute SAH with a ruptured aneurysm on the anterior communicating artery (Fisher grade 3). On bleed day 6, she developed a sudden onset of global aphasia and left hemiparesis preceded by cEEG changes consistent with vasospasm. A stat chemical dilator therapy was performed and she recovered without significant neurological deficits. A real-time and protocol-based cEEG can be utilized in order to avoid any delay in detection of vasospasm in aneurysmal SAH and thereby improve clinical outcomes.

Key Words : Continuous electroencephalography · Vasospasm · Delayed cerebral ischemia · Subarachnoid hemorrhage · Transcranial doppler · Cerebral aneurysm.

\section{INTRODUCTION}

In aneurysmal subarachnoid hemorrhage (SAH), symptomatic vasospasm, and its associated delayed cerebral ischemia (DCI), are not uncommon, and often result in significant morbidity and mortality ${ }^{5}$; however, a timely recognition of ischemic insults and the prompt use of interventions such as stat chemical vasodilators or balloon angioplasty are critical in order to prevent irreversible cerebral damage ${ }^{9)}$. We present a case of successful and prompt detection of a symptomatic vasospasm using real-time continuous electroencephalograpy (cEEG). This case has resulted in avoiding any permanent neurologic deficits for the patient.

\section{CASE REPORT}

A 50-year-old woman without any significant medical history presented with a sudden-onset of severe bifrontal headache. She arrived at the emergency room within the first 2 hours of symptom onset. Initially, she was only mildly drowsy (Hunt
Hess grade III) and had a Glasgow Coma Scale score of 13. Initial brain computed tomography (CT) and CT angiography (CTA) showed a diffuse subarachnoid hemorrhage (SAH) in the basal cistern and bilateral sylvian fissures with thick hemorrhages without intraventricular hemorrhage (Fisher grade 3 ), and a wide-neck aneurysm of $7.3 \times 6 \mathrm{~mm}$ at the anterior communicating artery (AcoA). The aneurysm was clipped immediately. Management of blood pressure and intracranial pressure was in accordance with the latest stroke guidelines at the neurological intensive care unit (ICU) (Fig. 1).

cEEG (Grass Technologies, West Warwick, RI, USA), and daily transcranial Doppler (TCD) were carried out from postbleed days 5 to 9 (with the day of the SAH as day 0 ) to detect vasospasm according to a predetermined cEEG protocol. Her cEEG revealed diffuse theta slowing without asymmetry and TCD mean flow velocities increased to $100 \mathrm{~cm} / \mathrm{s}$ and $106 \mathrm{~cm} / \mathrm{s}$ in the right and left middle cerebral arteries (MCA) respectively, resulting in respective Lindegaard ratios of 3.03 and 3.31 on post-bleed day 5 .

On post-bleed day 6 , the patient's level of consciousness re-

- Received : November 7, 2014 • Revised : February 4, 2015 •Accepted : February 23, 2015

- Address for reprints : Moon-Ku Han, M.D., Ph.D.

Department of Neurology, Seoul National University Bundang Hospital, 82 Gumi-ro 173beon-gil, Bundang-gu, Seongnam 13620, Korea

Tel : +82-31-787-7464, Fax : +82-31-787-4059, E-mail : mkhan@snu.ac.kr

- This is an Open Access article distributed under the terms of the Creative Commons Attribution Non-Commercial License (http://creativecommons.org/licenses/by-nc/3.0) which permits unrestricted non-commercial use, distribution, and reproduction in any medium, provided the original work is properly cited. 
covered completely, and was stable to be transferred out of the neurological ICU. Her neurological status was stable during the daily morning round ( 9 a.m.) and the hospital's visiting hours (10 : 30 a.m.). Her cEEG alpha/delta ratio (ADR) was not re- markable at 10 a.m. From then on, there was a significant ADR abnormalitiy, and the neurointensivist detected a progressive and rapid decrement of the ADR on the left side (more in C3$\mathrm{P} 3$ than in F3-C3) compared to the right at 11: 45 a.m. The de-
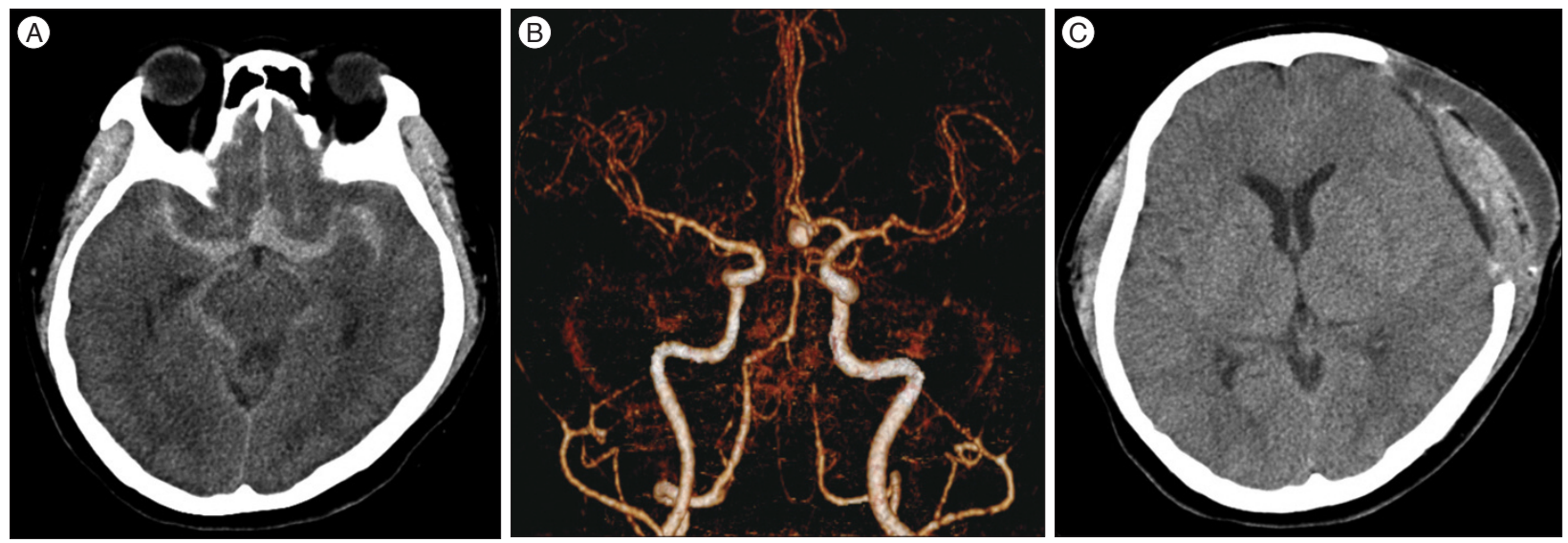

Fig. 1. Brain CT and CTA. Initial brain CT (A) and CTA (B) showing a diffuse subarachnoid hemorrhage without intraventricular hemorrhage (Fisher grade 3) and a wide-neck aneurysm of $7.3 \times 6 \mathrm{~mm}$ at the anterior communicating artery. Follow-up CT on post-bleed day 11 (C) shows mild epi- and sub-dural hematoma but no ischemic stroke.

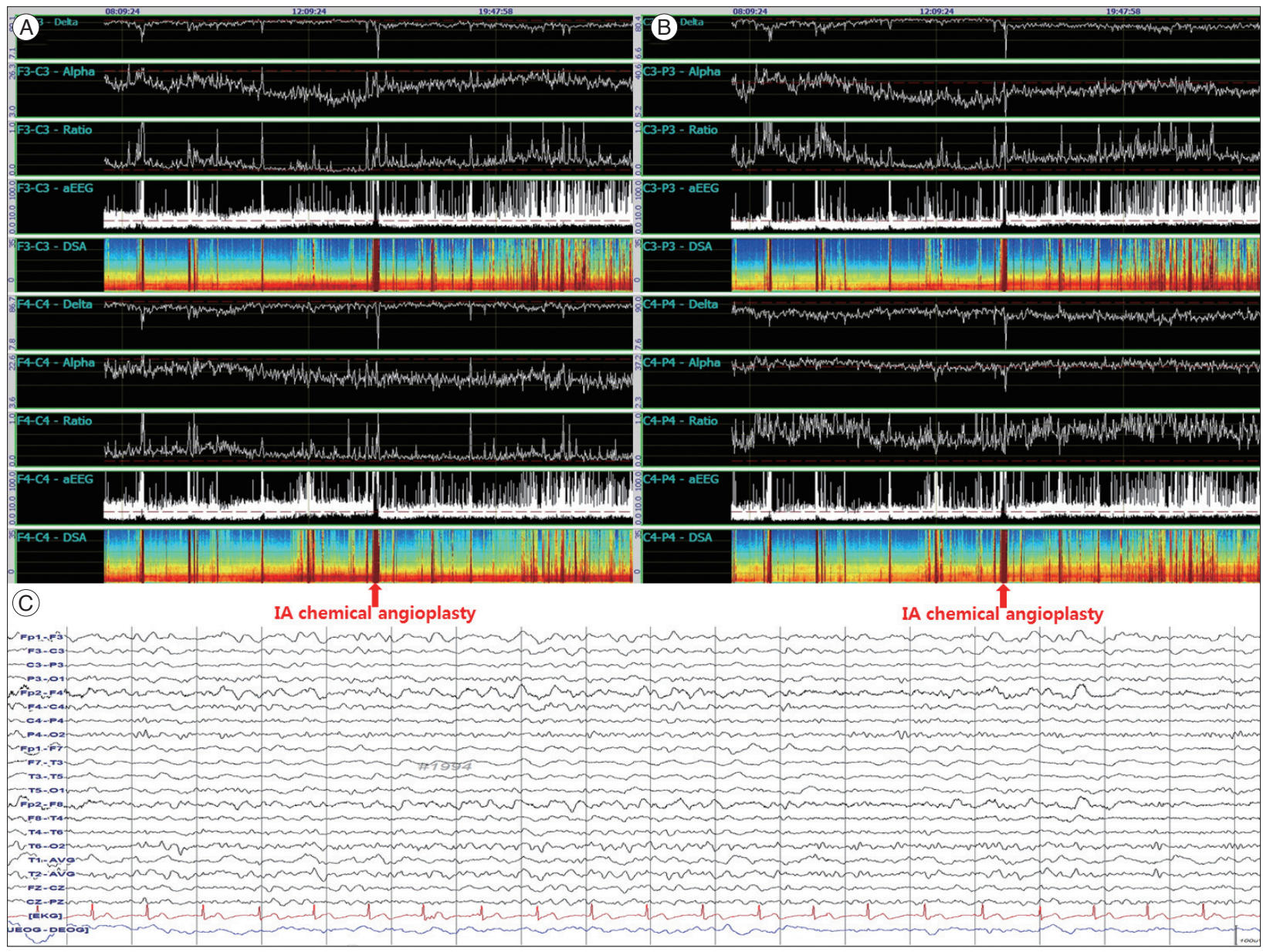

Fig. 2. Quantitative continuous EEG. Quantitative continuous EEG data from two bipolar channels in the bilateral fronto-central (F3-C3, F4-C4) and centro-parietal (C3-P3, C4-P4) regions showing progressive decrement of the alpha-delta ratio after 10 a.m. on the left hemisphere with more prominence on the centro-parietal than fronto-central region, and an increase of alpha-delta ratio after intra-arterial chemical angioplasty (A and B). Raw EEG data just before angioplasty, showing the rise in asymmetry with delta slowing on the left hemisphere (C). Ratio : alpha-to-delta ratio, DSA : density spectral array, EEG : electroencephalogram, aEEG : amplitude EEG. 

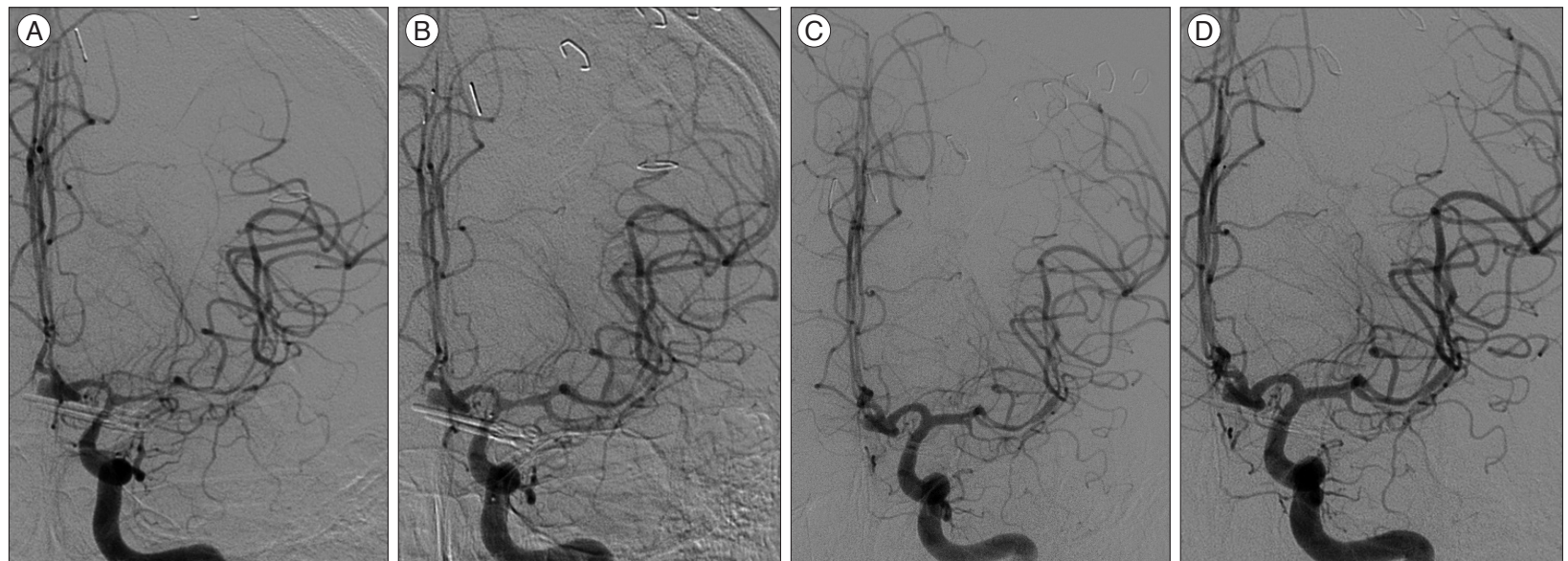

Fig. 3. Digital subtraction angiography before and after chemical angioplasty. On post-bleed day 6 , a first conventional angiography before chemical angioplasty $(\mathrm{A})$ shows severe vasospasm in the anterior cerebral artery and middle cerebral artery of the left hemisphere and a residual sac on the clipped aneurysm. Conventional angiography after chemical angioplasty with $4 \mathrm{mg}$ nimodipine (B) shows an increase in middle cerebral artery caliber. On post-bleed day 8, final conventional angiography before chemical angioplasty $(\mathrm{C})$ shows that moderate vasospasm on the left middle cerebral artery remains, and that vasospasm improves after chemical angioplasty with $5 \mathrm{mg}$ nimodipine (D).

crease of ADR was more than 60\% change from baseline (Fig. 2). Shortly after this, the patient became acutely right hemiparetic (Medical Research Council grade 3/5) with global aphasia. We attributed this worsening as symptomatic vasospasm in the MCA territory based on quantitative cEEG and neurological examinations. During preparation for a corrective procedure, stat TCD was performed and the mean flow of the left MCA was shown to be further increased $(161 \mathrm{~cm} / \mathrm{s})$ with a Lindegaard ratio of 5.03. Stat conventional cerebral angiography without CT angiography or CT perfusion was performed, and it showed severe vasospasm in the anterior cerebral artery (ACA) and MCA of the left hemisphere and there was a residual sac on the clipped aneurysm (Fig. 3). Successful chemical vasodialtion with intra-arterial (IA) infusion of $4 \mathrm{mg}$ of nimodipine was carried out.

The patient's neurological deficits including her moderate motor aphasia and leg weakness on the right side remained in the period immediately after the procedure; however, they were markedly improved compared to their severity before the procedure. On post-bleed day 7, her neurological status improved, but she still had minimal motor aphasia and leg weakness.

On post-bleed day 8 , the mean flow velocity of the left MCA was markedly increased to $207 \mathrm{~cm} / \mathrm{s}$ with a 6.27 Lindegaard ratio in the morning. There was no asymmetry of ADR on cEEG and no symptom worsening. Although the patient's vasospasm was asymptomatic, we decided to perform conventional angiography because of the high probability of its change into symptomatic vasospasm. The moderate vasospasm remained stationary. IA angioplasty with $5 \mathrm{mg}$ of nimodipine was performed again. Finally, she was discharged to home with only mild dysarthria after successful re-clipping of her AcoA aneurysm.

\section{DISCUSSION}

High-grade SAH has a high risk of developing vasospasm and DCI, from which secondary brain injury may develop, and result in death ${ }^{2)}$. However, it is not possible to predict when or if vasospasm after SAH will occur and this reality makes the condition difficult to treat. Although daily TCD testing might help to detect vasospasm, the role of TCD as a real-time detection tool has been criticized for its poor temporal resolution ${ }^{10)}$.

The goal of neuromonitoring in neurological ICU is to forestall secondary brain injury as soon as possible, and to prevent permanent injury by triggering timely interventions. cEEG serves this purpose well. A previous study reported that ADR demonstrated the strongest association with DCI compared with other indices and clinically useful cut-offs were defined 6 consecutive clip (one clip defined as 1 minute) with a $>10 \%$ decrease in ADR from baseline and any single clip with a $>50 \%$ as reasonable values ${ }^{1)}$. This association was based on EEG changes correlating with the degree of neuronal injury and cerebral blood flow impairment ${ }^{4,6)}$. The onset of clinical symptoms due to vasospasm after aneurysmal SAH may be preceded by decrement and asymmetry of ADR on cEEG, which can be detected in real time $e^{1)}$.

Since July 2013, our cEEG protocol for vasospasm has been applied in cases of high grade aneurysmal SAH and its summary is as follows : 1) cEEG monitoring is performed using a 21-electrode longitudinal bipolar montage according to the international 10-20 system. 2) The real-time quantitative cEEG (ADR, alpha power, delta power, amplitude EEG, and density spectral array) is checked at least every 2 h. 3) The focus is on F3-C3-F4-C4 and C3-P3/C4-P4 in AcoA aneurysm, on C3-P3/ C4-P4 in MCA-bifurcation aneurysm, and on C3-P3/C4-P4 and $\mathrm{P} 3-\mathrm{O} 1 / \mathrm{P} 4-\mathrm{O} 2$ in aneurysm of the posterior communicating $\operatorname{artery}^{1)}$.

The interval from the last normal period to the detection time was about 75 minutes, which might be attributable to the cEEG monitoring protocol. The time from detection to IA angioplasty was 115 minutes. Conventional angiography with 
chemical angioplasty was always available in our hospital but, obtaining procedural consent from her family was somewhat delayed because they were not always present outside of the hospital's visiting hours. As for vasospasm in aneurysmal SAH, rapid treatment within a 2-hour window may be profitable for promoting not only angiographic but also clinical improvements $^{7,9)}$. We believe that this protocol-based treatment may lead to a rapid clinical response, which has facilitated the complete recovery of this particular patient.

In this case, routine TCD before symptom onset did not predict vasospasm because of the impossibility of continuous monitoring, and there did not appear to be a relationship between clinical symptoms, MCA flow, and Lindegaard ratio after cerebral angioplasty (post-bleed day 8). TCD detects high mean cerebral blood flow velocities in the major vessels. The flow velocities are dependent on the vessels' diameter and insensitive to vasospasm affecting distal vasculature ${ }^{8)}$. Moreover, hypervolemia can lead to high velocities, which increases the false-positive rate without having true DCI due to cerebral vasospasm. In addition, TCD can be very limited in patients with poor acoustic windows. Detecting a perfusion defect rather than a decrease in vessel caliber is more indicative of $\mathrm{DCI}^{3)}$. However, perfusion studies such as CT perfusion cannot provide continuous data.

\section{CONCLUSION}

Real-time cEEG as well as daily routine TCD may help to detect vasospasm in aneurysmal SAH. Further, a protocol-based treatment algorithm may also reduce the permanent ischemic insult by reducing onset-to-detection time. Multidisciplinary care including neurointerventionist, neurointensivist, neurosurgeon, neurologist and continuous monitoring could dramatically improve the clinical outcomes of patients with aneurysmal SAH.

\section{References}

1. Claassen J, Hirsch LJ, Kreiter KT, Du EY, Connolly ES, Emerson RG, et al. : Quantitative continuous EEG for detecting delayed cerebral ischemia in patients with poor-grade subarachnoid hemorrhage. Clin Neurophysiol 115 : 2699-2710, 2004

2. Connolly ES Jr, Rabinstein AA, Carhuapoma JR, Derdeyn CP, Dion J, Higashida RT, et al. : Guidelines for the management of aneurysmal subarachnoid hemorrhage : a guideline for healthcare professionals from the American Heart Association/American Stroke Association. Stroke 43 : 1711-1737, 2012

3. Dankbaar JW, de Rooij NK, Velthuis BK, Frijns CJ, Rinkel GJ, van der Schaaf IC : Diagnosing delayed cerebral ischemia with different CT modalities in patients with subarachnoid hemorrhage with clinical deterioration. Stroke 40 : 3493-3498, 2009

4. Foreman B, Claassen J : Quantitative EEG for the detection of brain ischemia. Crit Care 16:216, 2012

5. Frontera JA, Claassen J, Schmidt JM, Wartenberg KE, Temes R, Connolly ES Jr, et al. : Prediction of symptomatic vasospasm after subarachnoid hemorrhage : the modified fisher scale. Neurosurgery 59 : 21-27; discussion 21-27, 2006

6. Jordan KG : Emergency EEG and continuous EEG monitoring in acute ischemic stroke. J Clin Neurophysiol 21 : 341-352, 2004

7. Kim SS, Park DH, Lim DJ, Kang SH, Cho TH, Chung YG : Angiographic features and clinical outcomes of intra-arterial nimodipine injection in patients with subarachnoid hemorrhage-induced vasospasm. J Korean Neurosurg Soc 52 : 172-178, 2012

8. Macdonald RL, Rosengart A, Huo D, Karrison T : Factors associated with the development of vasospasm after planned surgical treatment of aneurysmal subarachnoid hemorrhage. J Neurosurg 99 : 644-652, 2003

9. Rosenwasser RH, Armonda RA, Thomas JE, Benitez RP, Gannon PM, Harrop J : Therapeutic modalities for the management of cerebral vasospasm : timing of endovascular options. Neurosurgery 44 : 975-979; discussion 979-980, 1999

10. Vespa PM, Nuwer MR, Juhász C, Alexander M, Nenov V, Martin N, et al. : Early detection of vasospasm after acute subarachnoid hemorrhage using continuous EEG ICU monitoring. Electroencephalogr Clin Neurophysiol 103 : 607-615, 1997 